OPEN ACCESS

Edited by:

Reto Asmis,

Wake Forest School of Medicine,

United States

Reviewed by:

Keyue Ding,

Queen's University, Canada

Irma Bisceglia,

San Camillo-Forlanini Hospital, Italy

*Correspondence:

Cezar lliescu

ciliescu@mdanderson.org

${ }^{\dagger}$ These authors have contributed equally to this work

Specialty section: This article was submitted to

Cardio-Oncology,

a section of the journal

Frontiers in Cardiovascular Medicine

Received: 12 October 2021 Accepted: 20 December 2021

Published: 14 February 2022

Citation:

Thomason N, Monlezun DJ, Javaid A,

Filipescu A, Koutroumpakis E,

Shobayo F, Kim P, Lopez-Mattei J,

Cilingiroglu M, lliescu $G$,

Marmagkiolis K, Ramirez PT and

lliescu C (2022) Percutaneous Coronary Intervention in Patients With

Gynecological Cancer: Machine

Learning-Augmented Propensity

Score Mortality and Cost Analysis for 383,760 Patients.

Front. Cardiovasc. Med. 8:793877. doi: 10.3389/fcvm.2021.793877

\section{Percutaneous Coronary Intervention in Patients With Gynecological Cancer: Machine Learning-Augmented Propensity Score Mortality and Cost Analysis for 383,760 Patients}

\author{
Nicole Thomason ${ }^{1+}$, Dominique J. Monlezun ${ }^{2,3 t}$, Awad Javaid ${ }^{4}$, Alexandru Filipescu ${ }^{1}$, \\ Efstratios Koutroumpakis ${ }^{2}$, Fisayomi Shobayo ${ }^{1}$, Peter Kim², Juan Lopez-Mattei ${ }^{2}$, \\ Mehmet Cilingiroglu ${ }^{4}$, Gloria lliescu ${ }^{5}$, Kostas Marmagkiolis ${ }^{6}$, Pedro T. Ramirez ${ }^{7}$ and \\ Cezar lliescu ${ }^{2 *}$ \\ ${ }^{1}$ Division of Cardiology, The University of Texas Health Sciences Center at Houston, Houston, TX, United States, \\ ${ }^{2}$ Department of Cardiology, The University of Texas M.D. Anderson Cancer Center, Houston, TX, United States, ${ }^{3}$ Center for \\ Artificial Intelligence \& Health Equities, Global System Analytics \& Structures, New Orleans, LA, United States, ${ }^{4}$ Department \\ of Internal Medicine, University of Nevada Las Vegas School of Medicine, Las Vegas, NV, United States, ${ }^{5}$ Department of \\ Internal Medicine, The University of Texas Health Sciences Center at Houston, Houston, TX, United States, ${ }^{6}$ Division of \\ Cardiovascular Disease, University of Arkansas for Medical Sciences, Little Rock, AR, United States, ${ }^{7}$ Department of GynOnc \\ and Reproductive Medicine, The University of Texas Health Sciences Center at Houston, Houston, TX, United States
}

Background: Despite the growing number of patients with both coronary artery disease and gynecological cancer, there are no nationally representative studies of mortality and cost effectiveness for percutaneous coronary interventions (PCl) and this cancer type.

Methods: Backward propagation neural network machine learning supported and propensity score adjusted multivariable regression was conducted for the above outcomes in this case-control study of the 2016 National Inpatient Sample (NIS), the United States' largest all-payer hospitalized dataset. Regression models were fully adjusted for age, race, income, geographic region, cancer metastases, mortality risk, and the likelihood of undergoing PCl (and also with length of stay [LOS] for cost). Analyses were also adjusted for the complex survey design to produce nationally representative estimates. Centers for Disease Control and Prevention (CDC)-based cost effectiveness ratio (CER) analysis was performed.

Results: Of the 30,195,722 hospitalized patients meeting criteria, 1.27\% had gynecological cancer of whom $0.02 \%$ underwent PCl including $0.04 \%$ with metastases. In propensity score adjusted regression among all patients, the interaction of $\mathrm{PCl}$ and gynecological cancer (vs. not having PCl) significantly reduced mortality $(\mathrm{OR} 0.53,95 \% \mathrm{Cl}$ $0.36-0.77 ; p=0.001$ ) while increasing LOS (Beta 1.16 days, 95\% Cl 0.57-1.75; $p<$ 0.001 ) and total cost (Beta $\$ 31,035.46,95 \% \mathrm{Cl} 26758.86-35312.06 ; p<0.001)$. Among gynecological cancer patients, mortality was significantly reduced by $\mathrm{PCl}$ (OR 0.58 , 95\% Cl 0.39-0.85; $p=0.006$ ) and being in East North Central, West North Central, South Atlantic, and Mountain regions (all $p<0.03$ ) compared to New England. $\mathrm{PCl}$ reduced 
mortality but not significantly for metastatic patients (OR $0.74,95 \% \mathrm{Cl} 0.32-1.71 ; p=$ 0.481). Eighteen extra gynecological cancer patients' lives were saved with PCl for a net national cost of $\$ 3.18$ billion and a CER of $\$ 176.50$ million per averted death.

Conclusion: This large propensity score analysis suggests that $\mathrm{PCl}$ may cost inefficiently reduce mortality for gynecological cancer patients, amid income and geographic disparities in outcomes.

Keywords: gynecologic malignancies, gynecological tumors, PCI, percutaneous coronary intervention, cardio oncology

\section{INTRODUCTION}

Cardiovascular disease (CVD) and cancer remain the two most common causes of mortality among non-communicable diseases in Western countries (1). The bidirectional relationship between the two, with cancer patients or survivors having a significant burden of CVD and patients with CVD posing an increase in cancer incidence, has become more evident over the last decade and is reflected by the heightened interest in the discipline of cardio-oncology $(2,3)$. Common risk factors such as tobacco use, poor diet, and chronic inflammatory state are implicated in both disease states (4). Cancer commonly induces a pro-thrombotic state, which can be compounded by side effects of surgical interventions, chemotherapy, radiotherapy, and immunotherapy $(3,5)$ and trigger cardiovascular events. The recent improvement in overall long-term survival of cancer patients (6), likely related to the progress in cancer therapies, has been paralleled by an increase in the number of percutaneous coronary interventions (PCI) performed in cancer patients (7). Knowing the prevalence of acute coronary syndrome (ACS) in the general population requiring PCI, CVD burden in these cancer patients appears to be vastly underestimated.

Treatment of ACS in cancer patients is challenging, as each type of cancer has a unique clinical presentation and underlying physiology that calls for personalized care. The primary organ site, stage, and presence of metastases are all modifying factors that can influence post-PCI outcomes. Historically this understanding has not been reflected in clinical practice, partly due to the exclusion of patients with cancer from cardiovascular clinical trials and vice versa $(8-10)$. While there is now limited data exploring the overall prognostic impact of cancer on PCI outcomes (11-14), there is no data regarding PCI outcomes in gynecological cancer patients. Reported incidence of gynecologic malignancies in the U.S. is approximately 94,000 cases per year (15), with the most common malignancy being uterine cancer $(26.82$ cases per 100,000$)$ and the least common vaginal cancer (0.66 per 100,000).

Gynecological cancer patients have special considerations when determining risk for ACS and potential intervention with PCI. Women with endometrial cancer, a population particularly characterized by significant rates of obesity and diabetes mellitus, have been found to have a 1.5 -fold increased 10-year risk of CVD when compared to the general population (16). As many as $22 \%$ of endometrial cancer patients present at diagnosis with three or more risk factors of coronary artery disease (CAD)
(16). Furthermore, death from CVD has been found to be more prevalent in patients with endometrial cancer (17). In women who have undergone debulking procedures for epithelial ovarian carcinoma, the highest risk for hospital readmission perioperatively is a cardiopulmonary event (18). Platinumbased chemotherapeutic agents are frequently utilized for the treatment of ovarian and cervical cancer and are associated with multiple cardiotoxic side effects, with such cardiotoxic drugs as anthracyclines (including doxorubicin and cyclophosphamide) being frequently used for recurrent ovarian cancer (19). While the safety of common cardiovascular interventions such as percutaneous coronary intervention (PCI) in gynecologic cancer patients is not well-described, coronary artery bypass grafting $(C A B G)$ is considered a relative contraindication in patients with cancer due to an increased risk of metastatic dissemination during extracorporeal circulation (20). To bridge this knowledge gap, we used a large contemporary national database and examined the outcomes and economics of revascularization procedures in patients with gynecologic malignancies, stratified by specific type of cancer and stage.

\section{METHODS}

We defined gynecologic cancer in this analysis as any cancer involving the female reproductive system and further classified it based on specific anatomic location, including cancers of the ovaries, cervix, uterus, vagina, and vulva.

\section{Data Source}

The data source for this study was the 2016 United States (U.S.) National Inpatient Sample (NIS) for hospital discharges, the largest all-payer inpatient dataset in the nation, sponsored by the U.S. Department of Health and Human Services' Agency for Healthcare Research and Quality and maintained within the Healthcare Cost and Utilization Project (HCUP). The NIS currently accounts for approximately 1 in 5 discharges from all community hospitals in the U.S. To reduce sampling bias, the sampling strategy has been modified in the most recent data to produce results more generalizable to all inpatient discharges in the country. In 2016, the NIS data coding adopted the International Classification of Diseases, Tenth Revision, Clinical Modification (ICD-10-CM). Diagnoses of cancer and CVD were maded up to and including the index hospitalization period per patient based on the reported ICD-10. Cardiotoxic oncological treatment both prior and active were not reported in the dataset. 


\section{Study Design}

This is the first nationally representative multicenter analysis of inpatient mortality and total cost among all eligible hospitalized adults with CAD by PCI (yes/no) and PCI and cancer (yes/no), including overall and by primary organ site. The 2016 NIS dataset was selected for this study as it is the among latest available datasets and the first to use ICD-10 coding and thus betterreflects current clinical trends in PCI use compared to prior available datasets. Study inclusion criteria was all NIS hospitalizations for adults age 18 years or older during 2016. This study used de-identified data and was conducted according to the ethical principles in the Declaration of Helsinki.

Subjects undergoing PCI were identified by the ICD-10 procedure codes of 00.66 (percutaneous transluminal coronary angioplasty), 36.06 [insertion of non-drug-eluting coronary artery stent(s)], or 36.07 [insertion of drug-eluting coronary artery stent(s)]. ICD-10 diagnosis were used to identify gynecological cancers: C540, C541, C542, C543, C548, C549, C55, D070, Z8542, C530, C531, C538, C539, D060, D061, D067, D069, R87610, R87611, R87612, R87613, R87614, Z8541, Z86001, C561, C562, C569, Z8543, C510, C511, C512, C518, C519, C52, C5700, C5701, C5702, C5710, C5711, C5712, C5720, C5721, C5722, C573, C574, C577, C578, C579, C58, D071, D072, D0730,D0739, R87620, R87621, R87622, R87623, R87624, Z8540, Z8544. ICD10 codes were used to identify demographics, comorbidities, and outcomes. HCUP tools such as the Clinical Classification Software, which had been used prior to the NIS 2016 dataset for such purposes as classifying cancer (e.g., by primary type and current vs. historical), were not used in this study because they were found by HCUP as a beta version to be unreliable when applied to the 2016 dataset's ICD-10 data.

\section{Bivariable Statistical Analysis}

Descriptive statistics for demographics and comorbidities were performed for the full sample. Comorbidities were selected for analysis (and identified in the dataset by their ICD-10 scores) based on their clinical and/or statistical significance for similar studies in the existing literature. The comorbidities included in this study were diabetes, hypertension, peripheral vascular disease, hyperlipidemia, smoking, obesity, poor diet, stroke, congestive heart failure, cardiac arrest, myocardial infarction, cardiogenic shock, valvular disease, HIV, alcohol abuse, opioid abuse, anemia, chronic obstructive pulmonary disease, coagulopathy, depression, cirrhosis, chronic kidney disease, and malignancy (overall and by primary malignancy type).

Bivariable sub-group analysis was then conducted among gynecological malignancy patients according to the following: (a) inpatient all-cause mortality (yes/no); (b) PCI (yes/no) among the overall sample, stratified by metastases (yes/no) and in subgroup analyses among patients with malignancy; (c) PCI vessel number (multi- vs. single-vessel); (d) malignancy (yes/no) in subgroup analyses among patients who died with non-ST segment elevation myocardial infarction (NSTEMI) and separately among those with ST segment elevation myocardial infarction (STEMI); (e) length of stay by gynecological malignancy type; (f) total cost by gynecological malignancy type. For continuous variables, independent sample $t$-tests were performed to compare means and Wilcoxon rank sum tests were performed for medians. For categorical variables, Pearson chi square tests or Fisher exact tests were performed to compare proportions.

\section{Regression Statistical Analysis}

To optimize the likelihood of validated and replicable results, the performance of the final multivariable regression models in sub-group analysis among gynecological malignancy patients was first assessed by backward propagation neural network machine learning by accuracy and root mean squared error (RMSE) to ensure they were comparable based on an integrated hybrid methodology of traditional statistics reinforced by machine learning $(21,22)$. Variables found to be statistically significant in the bivariable analysis were included in forward and backward stepwise regression to augment decision-making on which variables should be included in the final multivariable regression models. This regression analysis adjusted for the PCI propensity score was conducted to assess the following outcomes: (a) inpatient all-cause mortality (by logistic) and (b) total hospital costs (by linear, adjusting with the additional variable of total allcause length of stay) using the predictor of the interaction term between PCI and malignancy (to provide separate estimates of the associations of mortality and PCI, mortality and malignancy, and mortality with PCI and malignancy). The regression models separately assessed these outcomes according to the following major predictors: (a) historical or active malignancy (yes/no), and gynecological malignancy type (uterus, cervical, ovarian, other). Sub-group analysis without propensity score adjustment was conducted separately according to history of CAD (additionally with stratified analysis by ACS and active or prior malignancy), active malignancy, prior malignancy, presenting diagnosis of ACS, NSTEMI, unstable angina, UA), and STEMI. All models adjusted for age, race, income, geographic region, metastases, and mortality risk by diagnosis-related group (DRG). Other variables were excluded based upon the machine learning analysis and diagnostic testing to produce the most clinically and statistically justifiable models.

Next, machine learning-backed propensity score-adjusted multivariable regression was conducted for mortality and controlled for age, race, income, presence of metastases, and mortality risk by diagnosis-related group in addition to the likelihood of undergoing PCI and the NIS weights accounting for the cluster sample data structure. The propensity score was then created for the likelihood of undergoing PCI [the treatment, utilizing the same above variables used in the final regression model to given the double propensity score adjustment method (23-25)], balance was confirmed among blocks, and then the propensity score was included in the final regression models as an adjusted variable. This causal inference approach (propensity score adjustment) was selected because it is a widely accepted methodology to reduce but not eliminate selection bias and the effect of confounding variables. Such competing causal inference approaches as fixed, random, and mixed effects were not appropriate, though these have the added advantage of reducing unobserved variable bias, 
because the dataset lacked adequate repeated hospitalizations from the same subjects. Propensity score adjustment was used rather than covariate adjustment without the propensity score to enable a more complicated propensity score model (i.e., able to test interactions and higher order terms to produce the best estimated probability of treatment assignment) without risking over-parameterizing while still permitting diagnostic analysis of the final models to be done to confirm superior performance to simple covariate adjustment without the propensity score. Finally, propensity score adjustment rather than competing propensity score techniques was used because of its superior performance in the appropriate context (confirmed by current statistical theory and adequate diagnostic quantitative testing of the final models in cardiovascular studies) $(23,24)$, and because its inclusion in the final regression models had sufficient performance confirmation the below diagnostic tests.

To modify the final models until optimal performance was achieved, performance was first assessed relative to results from backward propagation neural network machine learning to ensure comparability by root mean squared error and accuracy. Regression model performance was additionally assessed with correlation matrix, area under the curve, HosmerLemeshow goodness-of-fit test, Akaike and Schwarz Bayesian information criterion, variance inflation factor, and tolerance, multicollinearity, and specification error.

The utility of this above hybrid analytic approach, which integrates the traditional statistical method of frequentist-based multivariable regression (supported by propensity score-based causal inference analysis) and supervised learning-based machine learning has been previously demonstrated, as causal inference results which are more familiar to medical science audiences can be confirmed and replicated automatically through machine learning (and thus may accelerate real-time findings on larger high-dimensional datasets as they already increasingly do for other economic sectors outside of medicine), while producing more rapid and accurate results compared to traditional statistics (25-30). An academic physician-data scientist and biostatistician (DJM) confirmed that the final regression models were sufficiently supported by the existing literature and clinical and statistical theory. Fully adjusted regression results were reported with $95 \%$ confidence intervals (CIs) with statistical significance set at a 2 -tailed $p$-value of $<0.05$.

\section{Cost Effectiveness Analysis}

Cost-effectiveness analysis was conducted according to the methodology detailed by the Centers for Disease Control and Prevention (cdc.gov/policy/polaris) and applied to PCI (intervention) vs. medical management alone (comparator): the net cost was calculated as the cost of implementation minuts the averted cost which then produced the ratio of net costs over change in health outcome or the cost-effectiveness ratio (CER), with a negative value in the ratio representing cost savings and a positive value indicating increased cost. The implementation cost was determined by the higher end of the cost of inpatient PCI taken from the National Cardiovacular Registry CathPCI Registry (31) and then multiplied by the number of procedures in the specified sub-group of cardiooncology patients below in this study's principle dataset (NIS). The averted cost was determined by the 2016 World Bank average life expectancy (worldbank.org/world-development-indicators) minutes the average 2016 NIS age in this study multiplied by the 2016 Quality Adjusted Life Year (\$50,000/year/patient) and the cases of mortality averted with the treatment vs. the comparator. The net national cost was calculated as the above implementation cost minus the averted cost. The CER was the above net national cost divided by the number of averted costs by the treatment vs. the comparator.

\section{Software}

Statistical analysis was performed with STATA 14.2 (STATACorp, College Station, Texas, USA), and machine learning analysis was performed with Java 9 (Oracle, Redwood Chores, California, USA).

\section{RESULTS}

\section{Descriptive Statistics and Bivariable Analysis}

Of the 30,195,722 hospitalized patients meeting criteria, 383,760 (1.3\%) had gynecological cancer. Among those, mean age was 63.3 years (standard deviation [SD] 15.7), 73.53\% were Caucasian, $38.07 \%$ had uterine cancer, $29.95 \%$ had cervical cancer, $29.51 \%$ had ovarian cancer, and $2.47 \%$ had other gynecological malignancy (Table 1). Out of the 383,760 patients with gynecological cancer, 7,215 (1.9\%) underwent PCI; of those who underwent PCI, 2,875 (39.8\%) had active malignancy and $460(6.4 \%)$ had metastases. Significantly patients with gynecological cancer vs. those without it underwent PCI (1.88 vs. $4.04 \%, p<0.001)$ even when matched by age and mortality risk as calculated by the NIS according to DRGs (2.35 vs. $5.52 \%, p<$ 0.001). Among patients receiving PCI, patients with vs. without gynecological were significantly less likely to have CAD (71.56 vs. $78.22 \%, p<0.001$ ) and presenting STEMI (10.24 vs. $15.09 \%, p<$ 0.001 ), but had comparable likelihood of diabetes, hypertension, and presenting NSTEMI.

A total of 794,147 (2.6\%) deaths were recorded, out of which 20,807 (2.6\%) were from gynecological malignancy (Table 1). Patients with gynecological cancer had significantly lower mortality when compared to non-gynecological cancer patients (2.30 vs. $4.54 \%, p=0.004)$. Furthermore, in patients with gynecological malignancy, mortality (yes/no) was significantly lower for Caucasian (69.09 vs. 73.65\%) but higher for African American patients (16.1 vs. 11.8\%) (both $p<0.001$ ) and those with metastases ( 54.5 vs. $22.6 \%, p<0.001$ ).

Among patients with gynecological malignancy, the median all-cause length of stay (LOS) was 3 days (range 2-6, $p$ $<0.001)$ and median cost of hospitalization in U.S. dollars was $34,657(18,894-62,952 ; p<0.001)$. The highest mortality (yes/no) percentage was ovarian vs. non-ovarian gynecoloical malignancy ( 0.60 vs. $0.37 \%$ ) followed by uterine vs. non-uterine (0.59 vs. $0.48 \%)$ (Table 2$)$. The longest mean LOS was ovarian cancer (5.39 days [SD 5.57]), followed by other gynecological malignancy (5.25 days [SD 7.87]), and the most expensive total 
TABLE 1 | Descriptive statistics and bivariable analysis by inpatient mortality $(N=$ 383,760 admissions).

\begin{tabular}{|c|c|c|c|c|}
\hline \multirow[t]{3}{*}{ Variables } & \multirow[t]{3}{*}{ Sample } & \multicolumn{2}{|c|}{ Inpatient mortality } & \multirow[t]{3}{*}{$P$-value } \\
\hline & & No & Yes & \\
\hline & & $\begin{array}{c}(373,1695 \\
97.38 \%)\end{array}$ & $\begin{array}{c}(10,065 ; \\
2.62 \%)\end{array}$ & \\
\hline
\end{tabular}

Demographics, No. (\%)

Age, years, mean (SD)

$63.31 \quad 63.19(15.71) \quad 67.82(13.82) \quad<0.001$

(15.68)

Race

All groups

White

Black

Hispanic

Asian

Native American

Other

Non-white

Income quartile

First

Second

Third

Fourth

Insurance

Type

Commercial

Medicare

Medicaid

VA

None

Non-commercial

Admission, No. (\%)

Non-elective

Weekend

Medical history

Diabetes

Hypertension

PVD

HLD

Obesity

Smoking

Poor diet

CVATIIA

$\mathrm{CHF}$

HFrEF

Exacerbation

Cardiac Arrest

Myocardial Infarction

STEMI

NSTEMI/UA

Cardiogenic shock

Valvular disease

HIV

$<0.001$

69.09

16.05

7.89

3.72

0.52

2.73

30.91

26.35

28.74

25.65

24.60

21.01

21.02

$<0.001$

25.24

25.30

23.12

58.96

11.38

4.49

2.05

76.88

74.76

74.70

73.00

18.42

72.55

18.26

89.44

24.64

19.72

58.97

3.43

29.11

12.67

0.60

0.05

6.66

5.66

2.14

6.51

13.91

5.86

2.19

3.78

2.48

5.02

0.20

$<0.001$

0.461

0.669

0.002

$<0.001$

0.002

0.320

$<0.001$

0.010

0.025

$<0.001$

$<0.001$

$<0.001$

$<0.001$

$<0.001$

$<0.001$

0.686

0.335
TABLE 1 | Continued

\begin{tabular}{lcccc}
\hline \multirow{2}{*}{ Variables } & Sample & \multicolumn{2}{c}{ Inpatient mortality } & \multirow{2}{*}{$\boldsymbol{P}$-value } \\
\cline { 3 - 4 } & & No & Yes & \\
\hline Alcohol abuse & 2.01 & 2.02 & 1.69 & 0.303 \\
Opioid abuse & 1.52 & 1.55 & 0.60 & 0.001 \\
Anemia & 29.90 & 29.61 & 40.64 & $<0.001$ \\
COPD & 15.27 & 15.26 & 15.45 & 0.819 \\
Coagulation disorder & 6.93 & 6.62 & 18.48 & $<0.001$ \\
Depression & 15.87 & 16.01 & 10.73 & $<0.001$ \\
Cirrhosis & 1.71 & 1.68 & 2.98 & $<0.001$ \\
\hline
\end{tabular}

$S D$, standard deviation; VA, Veteran Affairs; PVD, peripheral vascular disease; $H L D$, hyperlipidemia; CVA, cerebrovascular disease; TIA, transient ischemia attack; CHF, congestive heart failure; HFrEF, heart failure with reduced ejection fraction; STEMI, ST segment elevation myocardial infarction, NSTEMI, non-ST segment elevation myocardial infarction; UA, unstable angina; HIV, human immunodeficiency virus; COPD, chronic obstructive pulmonary disease; $C K D$, chronic kidney disease; $P C l$, percutaneous coronary intervention; $C A B G$, coronary artery bypass graft.

TABLE 2 | Summary bivariable outcome results by malignancy $(N=383,760$ admissions).

\begin{tabular}{|c|c|c|c|c|}
\hline \multirow[t]{3}{*}{ Malignancy } & \multicolumn{4}{|c|}{ Outcomes } \\
\hline & \multicolumn{2}{|c|}{ Mortality, No. (\%)* } & \multirow{2}{*}{$\begin{array}{l}\text { LOS, days, } \\
\text { mean (SD)** }\end{array}$} & \multirow{2}{*}{$\begin{array}{l}\text { Cost, USD, } \\
\text { mean (SD)** }\end{array}$} \\
\hline & No & Yes & & \\
\hline Gynecological & 1.27 & 1.52 & $5.03(5.71)$ & $\begin{array}{c}52925.20 \\
(69153.44)\end{array}$ \\
\hline Uterus & 0.48 & 0.59 & $4.99(5.57)$ & $\begin{array}{c}53907.51 \\
(69559.61)\end{array}$ \\
\hline Cervix & 0.38 & 0.30 & 4.74 (5.51) & $\begin{array}{c}48644.10 \\
(64795.88)\end{array}$ \\
\hline Ovarian & 0.37 & 0.60 & $5.39(5.57)$ & $\begin{array}{c}56708.13 \\
(72440.59)\end{array}$ \\
\hline Other & 0.09 & 0.07 & $5.25(7.87)$ & $\begin{array}{c}52326.71 \\
(67357.82)\end{array}$ \\
\hline
\end{tabular}

LOS, length of stay; SD, standard deviation; USD, US dollars; ${ }^{*} p<0.05$ for mortality (yes

vs. no); ${ }^{*} p<0.05$ for malignancy (yes/no).

(Continued) hospitalization cost was ovarian (USD 56,708 [SD 72440.59]) followed by uterine (53907.51 [SD 69559.61]).

\section{Multivariable Regression}

In propensity score-adjusted regression among all patients, the interaction of PCI and gynecological cancer (vs. not having PCI) was associated with significantly reduced mortality (OR $0.53,95 \%$ CI $0.36-0.77 ; p=0.001$; marginal effects likelihood: $-0.87 \%)$. Among gynecological cancer patients, mortality was similarly significantly reduced by PCI (OR $0.58,95 \%$ CI $0.39-$ $0.86 ; p=0.007$ ) as well as hospitalization in East North Central, West North Central, South Atlantic, and Mountain regions (all $p$ $<0.05)$ compared to New England. PCI reduced mortality but not significantly for patients with metastatic cancer (OR 0.74, 95\%CI 0.31-1.75; $p=0.493$ ) (Table 3). There were no significant racial or income disparities. 
TABLE 3 | Machine learning-augmented propensity score adjusted multivariable regression of inpatient mortality among gynecological malignancy patients ( $N=$ 383,760 admissions).

\begin{tabular}{|c|c|}
\hline Variable & OR (95\% Cl; $P$-value) \\
\hline Age by 10 years & $1.00(0.99-1.00 ; p=0.136)$ \\
\hline Non-white race & $1.22(1.08-1.36 ; p=0.001)$ \\
\hline \multicolumn{2}{|l|}{ Region } \\
\hline Mid-Atlantic & $0.95(0.73-1.24 ; p=0.715)$ \\
\hline East North Central & $0.73(0.56-0.95 ; p=0.021)$ \\
\hline West North Central & $0.71(0.50-0.99 ; p=0.044)$ \\
\hline South Atlantic & $0.75(0.57-0.98 ; p=0.038)$ \\
\hline East South Central & $1.04(0.73-1.48 ; p=0.830)$ \\
\hline West South Central & $1.03(0.76-1.36 ; p=0.851)$ \\
\hline Mountain & $0.61(0.42-0.88 ; p=0.008)$ \\
\hline Pacific & $0.99(0.76-1.29 ; p=0.935)$ \\
\hline \multicolumn{2}{|l|}{ Zip code income } \\
\hline 1st quartile & Reference \\
\hline 2nd quartile & $0.94(0.82-1.08 ; p=0.416)$ \\
\hline 3rd quartile & $0.89(0.78-1.03 ; p=0.124)$ \\
\hline 4th quartile & $0.87(0.75-1.02 ; p=0.086)$ \\
\hline $\mathrm{PCl}$ & $0.58(0.39-0.86 ; p=0.007)$ \\
\hline \multicolumn{2}{|l|}{ Malignancy } \\
\hline Metastases & $2.03(1.84-2.24 ; p<0.001)$ \\
\hline Mortality risk by DRG & $7.12(6.54-7.75 ; p<0.001)$ \\
\hline
\end{tabular}

OR, odds ratio; $\mathrm{Cl}$, confidence interval; $\mathrm{PCl}$, percutaneous coronary intervention; $D R G$, diagnosis-related group. The bold values are statistically significant.

In sub-group analysis by individual gynecological malignancy type, PCI significantly decreased all-cause mortality for uterine cancer (OR 0.49, 95\% CI 0.25-0.96; $p=0.038$ ) but not ovarian, cervix, or other. In sub-group analysis by ACS (including separately NSTEMI/UA vs. STEMI) and active malignancy (yes/no) among gynecological malignancy patients, PCI reduced mortality for all sub-groups but only significantly for patients with non-ACS active malignancy patients (OR 0.37, 95\% CI 0.15$0.89 ; p=0.027)$ and NSTEMI/UA prior malignancy patients (OR 0.19 , 95\%CI $0.05-0.72 ; p=0.014$ ) (Figure 1). In subgroup analysis by gynecology cancer by primary organ site and cancer status (without metastasis, with metastasis, and historical diagnosis all vs. no cancer), the highest mortality reductions with PCI were for patients with ovarian metastasis (Figure 2).

\section{Cost Effectiveness}

In propensity score adjusted regression among all patients, the interaction of PCI and gynecological cancer (vs. not having PCI) significantly increased LOS (Beta 1.16 days, 95\% CI 0.57-1.75; $p<0.001$ ) and total cost of stay (Beta $\$ 31035.46,95 \%$ CI 26758.86-35312.06; $p<0.001)$. Of the 7,215 gynecological cancer patients who underwent inpatient PCI, $0.25 \%$ or 18 extra gynecological cancer patients' lives were saved with PCI for a net national cost of $\$ 3.18$ billion and a cost effectiveness ratio (CER) of $\$ 176.50$ million per averted death.

\section{DISCUSSION}

Our study demonstrated that inpatient PCI can be safely performed in patients with gynecological cancer, including those with metastatic disease, albeit with increased cost and length of stay amid significant geographic disparities in mortality. This is the first known nationally representative, comprehensive machine learning-augmented, propensity score analysis of mortality and cost for patients with gynecological cancer vs. non-gynecological cancer patients in terms of PCI vs. medical management (including overall and by ACS).

Our analysis reveals that PCI does not increase mortality in patients with gynecologic cancer, regardless of the unique risks in this population. When analyzed by specific type of malignancy, PCI significantly reduced mortality for uterine cancer, while ovarian, cervical, and other gynecologic cancers had a nonstatistically significant reduction in mortality. This may at least be in part because patients with uterine cancer in contrast to the other gynecological cancers in this dataset had greater CVD risk factors (i.e. older with higher prevalence of hypertension and diabetes) and thus may be positioned to best benefit from PCI. The lack of increased mortality rate across all cancer types is likely not just statistical in nature and could suggest that routine/standard of care PCI if applied to this patient population would not translate in a significant increase in mortality.

Furthermore, this analysis shows that even when patients with gynecological vs. non-gynecological cancer have comparable age and mortality risk, they undergo PCI significantly less than patients without this cancer type, suggesting that inpatient PCI may be withheld from these patients (further research is required to clarify the reasons why which likely are multifactorial and can include lower clinical suspicion or more non-specific symptoms for CVD given typically younger age and less CVD risk factors). This finding was consistent across a wide range of age and mortality risk groups. While PCI may be offered less to cancer patients due to concerns of safety and efficacy, previous literature indicates that PCI is safe and beneficial in such population (11-14), and our real-world analysis shows PCI is safe to perform in gynecological cancer patients as well. The results presented here should promote the inclusion of patients with gynecological cancer undergoing cancer treatment and with acceptable medium- and long-term survival (least 6 months and preferably 1 year expected $>50 \%$ survival) in future cardiovascular trials and encourage physicians to more frequently utilize PCI in this patient population.

Other factors worth considering in future analyses are the type of stent used and medication used in gynecologic cancer patients. Standard balloon angioplasty or percutaneous old balloon angioplasty (POBA) has been shown to have overall worse outcomes compared to drug-eluting stents in the general population, and was considered a possible option in gynecologic cancer patients as the reduced duration of aspirin and Plavix or dual antiplatelet therapy (DAPT) with POBA may be beneficial to patients with an increased bleeding risk (32). Evolution of stent platforms, polymers and eluting medications over the last decade has translated in an abbreviated DAPT course, for certain indications (stable angina, abnormal stress 


\section{Fully adjusted odds ratio}

1.2

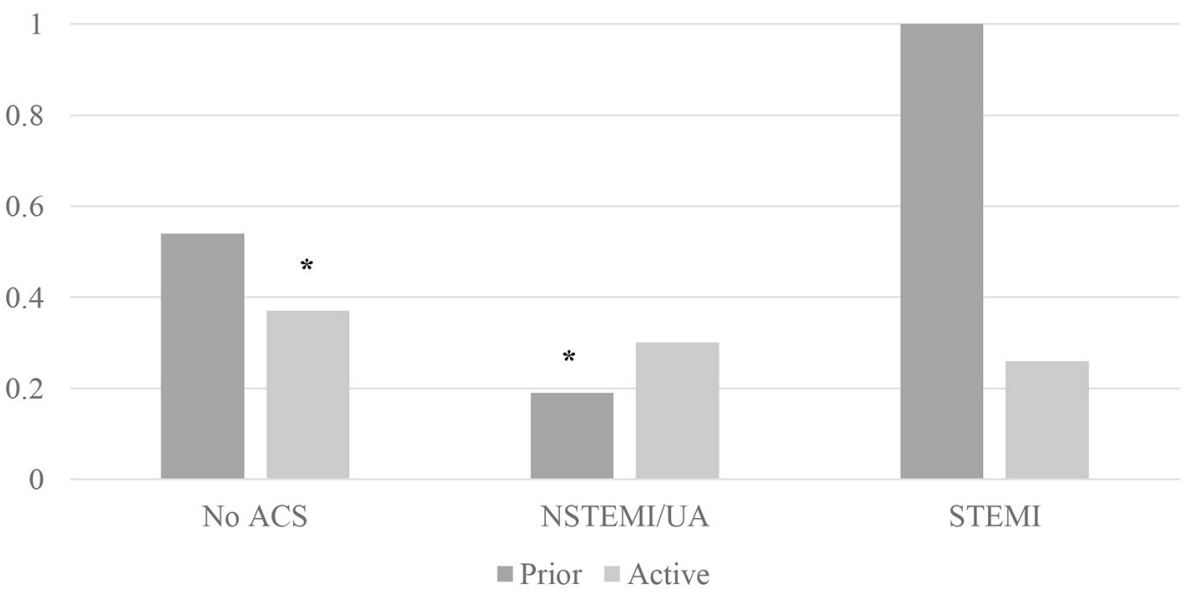

FIGURE 1 | Machine learning-augmented propensity score adjusted multivariable regression of inpatient mortality among gynecological malignancy patients ( $N$ = 383,760 admissions). Multivariable regression fully adjusted for age, race, income, metastases, and mortality risk by Diagnosis Related Group; NSTEMI/UA, non-ST elevation myocardial infarction/unstable angina; STEMI, ST-elevation myocardial infarction; ${ }^{\star} p<0.05$.

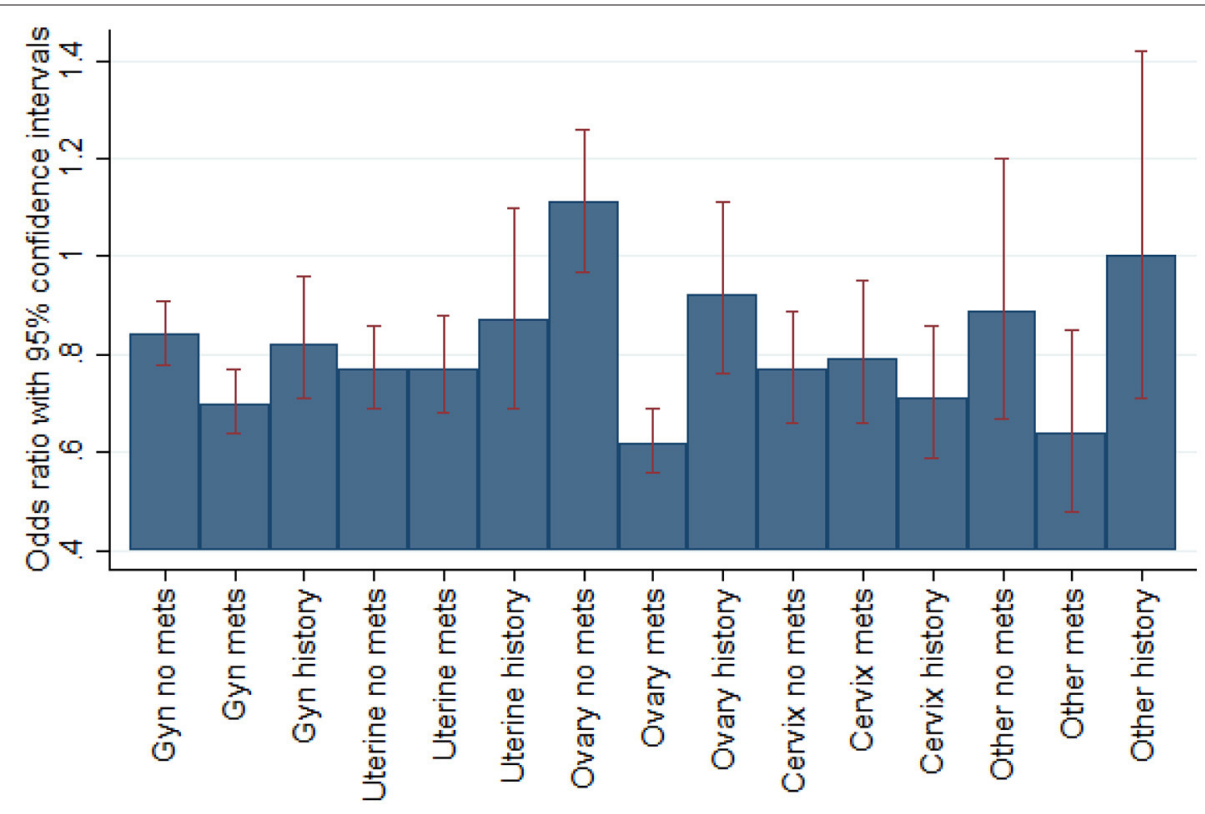

Cancer status

FIGURE 2 | Multivariable regression of mortality by gynecological oncology status vs. no cancer ( $N=383,760$ admissions). Fully adjusted for age, race, income, region, $\mathrm{PCI}, \mathrm{PCl}$ likelihood, and NIS-calculated mortality risk by DRG.

test) several stent have been approved for 1-3 months of DAPT. Patients with metastatic disease would require additional stratification that impacts decision making in these complex clinical challenges.

Our results should be interpreted with caution in the context their limitations, which include a non-randomized design with administrative data limited to inpatient variables without longitudinal individual follow-up data, particularly 3-month and 12-month mortality which can affect cost-effectiveness analysis. This study sought to overcome such limitations on its external and internal validity by utilizing multicenter nationally representative data with robust causal inference analysis to allow for the most reliable and reproducible results possible for this nuanced clinical topic. 


\section{CONCLUSION}

This study provides evidence that the clinical benefit of PCI may be safely extended to gynecological cancer patients, albeit with an increase in cost. There is also evidence of mortality disparity by geography and PCI underutilization in gynecological cancer patients despite clinical indication. This first known granular sub-group analysis by malignancy type, and active vs. prior cancer status suggests PCI significantly decreases mortality by type of gynecological cancer.

\section{REFERENCES}

1. Ferlay J, Steliarova-Foucher E, Lortet-Tieulent J, Rosso S, Coebergh JWW, Comber $\mathrm{H}$, et al. Cancer incidence and mortality patterns in Europe: Estimates for 40 countries in 2013. Eur J Cancer. (2015) 49:1374403. doi: 10.1016/j.ejca.2012.12.027

2. van Kruijsdijk RC, van der Graaf Y, Koffijberg H, de Borst GJ, Nathoe HM, Jaap Kappelle L, et al. Cause-specific mortality FL, and years of life lost in patients with different manifestations of vascular disease. Eur J Prevent Cardiol. (2015) 23:160-9. doi: 10.1177/20474873145 66998

3. Hooning MJ, Aleman BMP, van Rosmalen AJM, Kuenen MA, Klijn JGM, Leeuwen FE. Cause-specific mortality in long-term survivors of breast cancer: a 25-year follow-up study. Int J Radiat Oncol Biol Phys. (2006) 64:108191. doi: 10.1016/j.ijrobp.2005.10.022

4. Bonura F, Di Lisi D, Novo S, D'Alessandro N. Timely recognition of cardiovascular toxicity by anticancer agents: a common objective of the pharmacologist, oncologist, and cardiologist. Cardiovasc Toxicol. (2012) 12:93-107. doi: 10.1007/s12012-011-9141-z

5. Hooning MJ, Botma A, Aleman BM. Long-term risk of cardiovascular disease in 10-year survivors of breast cancer. J Natl Cancer Inst. (2007) 99:36575. doi: 10.1093/jnci/djk064

6. Henley BSJ, Singh SD, King J, Wilson R, Ryerson AB. Invasive cancer incidence and survival in the United States. Oncol Times. (2011) 64:75-6. doi: 10.15585/mmwr.mm6449a126

7. Potts JE, Iliescu CA, Lopez Mattei JC, Martinez SC, Holmvang L, Ludman $\mathrm{P}$, et al. Percutaneous coronary intervention in cancer patients: a report of the prevalence and outcomes in the United States. Eur Heart J. (2019) 40:1790-800. doi: 10.1093/eurheartj/ehy769

8. Mehta SR, Yusuf S, Peters RJ. Clopidogrel in unstable angina to prevent recurrent events trial (CURE) investigators. Treatment and outcomes of acute coronary syndrome in the cancer population. Eur Heart J. (2000) 21:203341. doi: 10.1053/euhj.2000.2474

9. Wallentin L, Becker RC, Budaj A, Cannon CP, Emanuelsson H, Held C. Ticagrelor versus clopidogrel in patients with acute coronary syndromes. N Engl J Med. (2009) 361:1045-57. doi: 10.1056/NEJMoa0 904327

10. Wiviott SD, Braunwald E, McCabe CH, Montalescot G, Ruzyllo W, Gottlieb S. Prasugrel versus clopidogrel in patients with acute coronary syndromes. $N$ Engl J Med. (2007) 357:2001-15. doi: 10.1056/NEJMoa0706482

11. Al-Hawwas M, Tsitlakidou D, Gupta N, Iliescu C, Cilingiroglu M, Marmagkiolis K. Acute coronary syndrome management in cancer patients. Curr. Oncol. Rep. (2018) 20:78. doi: 10.1007/s11912-018-0724-8

12. Iliescu C, Grines CL, Herrmann J, Yang EH, Cilingiroglu M, Charitakis K. SCAI Expert consensus statement: evaluation, management, and special considerations of cardio-oncology patients in the cardiac catheterization laboratory (endorsed by the cardiological society of india, and sociedad Latino Americana de Cardiologia intervencionista). Catheter Cardiovasc Interv. (2016) 87:E202-23. doi: 10.1002/ccd.26375

13. Iliescu C, Balanescu DV, Donisan T, Giza DE, Muñoz Gonzalez ED, Cilingiroglu M. Safety of diagnostic and therapeutic cardiac catheterization in cancer patients with acute coronary syndrome and chronic thrombocytopenia. Am J Cardiol. (2018) 122:146570. doi: 10.1016/j.amjcard.2018.07.033

\section{DATA AVAILABILITY STATEMENT}

The original contributions presented in the study are included in the article/supplementary material, further inquiries can be directed to the corresponding author/s.

\section{AUTHOR CONTRIBUTIONS}

All authors listed have made a substantial, direct, and intellectual contribution to the work and approved it for publication.

14. Giza DE, Marmagkiolis K, Mouhayar E, Durand JB, Iliescu C. Management of CAD in patients with active cancer: the interventional cardiologists' perspective. Curr. Cardiol. Rep. (2017) 19:56. doi: 10.1007/s11886-017-0862-x

15. Centers for Disease Control and Prevention. Gynecologic Cancer Incidence, United States-2012-2016. Atlanta, GA: Centers for Disease Control and Prevention, US Department of Health and Human Services (2019).

16. Kitson SJ, Lindsay J, Sivalingam VN, Lunt M, Ryan NAJ, Edmondson RJ, et al. The unrecognized burden of cardiovascular risk factors in women newly diagnosed with endometrial cancer: a prospective case control study. Gynecol Oncol. (2018) 148:154-60. doi: 10.1016/j.ygyno.2017.11.019

17. Felix AS, Bower JK, Pfeiffer RM, Raman SV, Cohn DE, Sherman ME, et al. High cardiovascular disease mortality after endometrial cancer diagnosis: Results from the Surveillance, Epidemiology, and End Results (SEER) Database. Int J Cancer. (2016) 140:555-64. doi: 10.1002/ijc.30470

18. Clark RM, Growdon WB, Wiechert A, Boruta D, Del Carmen M, Goodman AK, et al. Patient, treatment and discharge factors associated with hospital readmission within 30 days after surgical cytoreduction for epithelial ovarian carcinoma. Gynecol Oncol. (2013) 130:407-10. doi: 10.1016/j.ygyno.2013.05.034

19. Dasari S, Bernard Tchounwou P. Cisplatin in cancer therapy: molecular mechanisms of action. Eur J Pharmacol. (2014) 740:364-78. doi: 10.1016/j.ejphar.2014.07.025

20. Pinto CA, Marcella S, August DA. Cardiopulmonary bypass has a modest association with cancer progression: a retrospective cohort study. BMC Cancer. (2013) 13:519. doi: 10.1186/1471-2407-13-519

21. Balanescu DV, Monlezun DJ, Donisan T, Boone D, Cervoni-Curet F, Palaskas $\mathrm{N}$, et al. A cancer paradox: machine-learning backed propensity-score analysis of coronary angiography findings in cardio-oncology. I Invasive Cardiol. (2019) 31:21-6.

22. Monlezun DJ, Dart L, Vanbeber A, Smith-Barbaro P, Costilla V, Samuel C. Machine learning-augmented propensity score-adjusted multilevel mixed effects panel analysis of hands-on cooking and nutrition education versus traditional curriculum for medical students as preventive cardiology: multisite cohort study of 3,248 trainees over 5 years. Biomed Res Int. (2018) 2018:5051289. doi: 10.1155/2018/5051289

23. D'Agostino RB. Propensity score methods for bias reduction in the comparison of a treatment to a nonrandomized control group. Stat Med. (1998) 17:226581. doi: 10.1002/(SICI)1097-0258(19981015)17:19<2265::AID-SIM918>3.0.CO;2-B

24. Elze MC, Gregson J, Baber U, et al. Comparison of propensity score methods and covariate adjustment: evaluation in 4 cardiovascular studies. J Am Coll Cardiol. (2017) 69:345-57. doi: 10.1016/j.jacc.2016.10.060

25. Monlezun DJ, Lawless S, Palaskas N, Peerbhai S, Charitakis K, Marmagkiolis $\mathrm{K}$, et al. Machine learning-augmented propensity score analysis of percutaneous coronary intervention in over 30 million cancer and non-cancer patients. Front Cardiovasc Med. (2021) 8:620857. doi: 10.3389/fcvm.2021.620857

26. Mathias TL, Albright KC, Boehme AK, Monlezun DJ, George AJ, Jones E, et al. The impact of myocardial infarction vs. pneumonia on outcome in acute ischemic stroke. J Cardiovasc Dis. (2014) 2:1-3.

27. Scullen TA, Monlezun DJ, Siegler JE, George AJ, Schwickrath M, El Khoury R, et al. Cryptogenic stroke: clinical consideration of a heterogeneous ischemic subtype. J Stroke Cerebrovasc Dis. (2015) 24:9939. doi: 10.1016/j.jstrokecerebrovasdis.2014.12.024 
28. Johnson KW, Torres Soto J, Glicksberg BS, et al. Artificial intelligence in cardiology. J Am Coll Cardiol. (2018) 71:266879. doi: 10.1016/j.jacc.2018.03.521

29. Chen JH, Asch SM. Machine learning and prediction in medicine-beyond the peak of inflated expectations. $N$ Engl J Med. (2017) 376:2507-9. doi: 10.1056/NEJMp17 02071

30. Obermeyer Z, Emanuel EJ. Predicting the future-big data, machine learning, clinical medicine. $N$ Engl J Med. (2016) 375:1216-9. doi: 10.1056/NEJMp1606181

31. Amin AP, Patterson M, House JA, Giersiefen H, Spertus JA, Baklanov DV, et al. Costs associated with access site and same-day discharge among medicare beneficiaries undergoing percutaneous coronary intervention: an evaluation of the current percutaneous coronary intervention care pathways in the United States. JACC Cardiovasc Interv. (2017) 10:342-51. doi: 10.1016/j.jcin.2016.11.049

32. Moriya, M, Ishiwata, S, Fujimoto $\mathrm{Y}$. Characteristics and trends of POBA in current DES era. Cardiovasc Interv Ther. (2015) 30:315-19. doi: $10.1007 /$ s12928-015-0316-6
Conflict of Interest: The authors declare that the research was conducted in the absence of any commercial or financial relationships that could be construed as a potential conflict of interest.

Publisher's Note: All claims expressed in this article are solely those of the authors and do not necessarily represent those of their affiliated organizations, or those of the publisher, the editors and the reviewers. Any product that may be evaluated in this article, or claim that may be made by its manufacturer, is not guaranteed or endorsed by the publisher.

Copyright (c) 2022 Thomason, Monlezun, Javaid, Filipescu, Koutroumpakis, Shobayo, Kim, Lopez-Mattei, Cilingiroglu, Iliescu, Marmagkiolis, Ramirez and Iliescu. This is an open-access article distributed under the terms of the Creative Commons Attribution License (CC BY). The use, distribution or reproduction in other forums is permitted, provided the original author(s) and the copyright owner(s) are credited and that the original publication in this journal is cited, in accordance with accepted academic practice. No use, distribution or reproduction is permitted which does not comply with these terms. 